\title{
Decision rules for determining terrestrial movement and the consequences for filtering high-resolution GPS tracks - A case study using the African Lion (Panthera leo)
}

Richard Michael Gunner ( $D$ 792021@swansea.ac.uk)

Swansea University https://orcid.org/0000-0002-2054-9944

Rory P Wilson

Swansea University College of Science

Mark D Holton

Swansea University College of Science

Phil Hopkins

Swansea University College of Science

Stephen H Bell

Queen's University Belfast School of Biological Sciences

Nikki J Marks

Queen's University Belfast School of Biological Sciences

Nigel C Bennett

University of Pretoria Department of Zoology and Entomology

\section{Sam Ferreira}

SANParks: South African National Parks

\section{Danny Govender}

SANParks: South African National Parks

\section{Pauli Viljoen}

SANParks: South African National Parks

\section{Angela Bruns}

SANParks: South African National Parks

O. Louis van Schalkwyk

Government of South Africa, Department of Agriculture, Forestry and Fisheries

\section{Mads F Bertelsen}

Center for Zoo and Wild Animal Health, Copenhagen Zoo

\section{Carlos M Duarte}

King Abdullah University of Science and Technology

\section{Martin C van Rooyen}

University of Pretoria Department of Zoology and Entomology 


\section{Craig J Tambling}

University of Fort Hare, Department of Zoology and Entomology

\section{Aoife Goppert}

Queen's University Belfast School of Biological Sciences

\section{Delmar Diesel}

Queen's University Belfast School of Biological Sciences

D. Michael Scantlebury

Queen's University Belfast School of Biological Sciences

\section{Research Article}

Keywords: Acceleration, Animal behaviour, Data filtering, Global Positioning System (GPS), Highresolution, Speed, Terrestrial movement

Posted Date: June 15th, 2021

DOI: https://doi.org/10.21203/rs.3.rs-600317/v1

License: (c) (i) This work is licensed under a Creative Commons Attribution 4.0 International License. Read Full License 


\section{Abstract}

The combined use of Global Positioning System (GPS) technology and motion sensors within the discipline of movement ecology has increased over recent years. This is particularly the case for instrumented wildlife, with many studies now opting to record parameters at high (infra-second) sampling frequency. However, the detail with which GPS loggers can elucidate fine-scale movement depends on the precision and accuracy of fixes, with accuracy (specifically, location error and fix success rate) being affected by signal reception. We hypothesised that animal behaviour was the main factor affecting fix inaccuracy (particularly for collar-mounted tags sampling at high frequency). In conjunction to this, inherent GPS positional noise ('jitter'), would be most apparent during GPS fixes for non-moving locations, thereby producing disproportionate error during rest periods.

A Movement Verified Filtering (MVF) protocol was constructed to compare GPS-derived speed data to dynamic body acceleration (DBA). This was collected by a simultaneously deployed tri-axial accelerometer, to provide a computationally quick method for identifying genuine travelling movement. This method was tested on 11 free-ranging lions ( Panthera leo ) within the Kgalagadi Transfrontier park in the Kalahari Desert, fitted with collar-mounted GPS units and tri-axial motion sensors (Daily Diary; DD) recording at 1 and $40 \mathrm{~Hz}$, respectively.

The findings support the hypothesis and show that distance moved estimates were, on average, overestimated by $>80 \%$ prior to GPS screening. We present the conceptual and mathematical protocols for screening fix inaccuracy within high resolution GPS datasets.

We demonstrate the importance that MVF has for avoiding inaccurate and biased estimates of movement and caution the accuracy of findings from previous studies that employed minimal GPS preprocessing. Throughout, we address the applicability of comparing fine-scale indices of GPS- and motion sensor-borne data in tandem to qualify animal behaviour.

\section{Introduction}

A popular method to determine terrestrial animal movement uses Global Positioning Systems (GPS), which enables long-term continuous spatial monitoring of wild animals without disturbing them [for reviews see; 1, 2-5]. This approach has led to broad applications, including examination of home ranges [6, 7], migratory routes [8-10], habitat use [11, 12], resource allocation [13, 14], activity budgets [15-17] as well as social interactions [18]. Since their inception, animal-borne GPS's have reduced considerably in mass and size, whilst data storage capacity, battery longevity and affordability have improved $[5,19,20]$. Consequently, scientists can now track animals as small as ca. $20 \mathrm{~g}$ songbirds (Seiurus aurocapilla) [21] at frequencies as high as $10 \mathrm{~Hz}$ [e.g., 22], providing so much detail of animal movement that even animal behaviour can often be inferred [23-25]. Such inference is, however, limited by fix precision, regardless of fix accuracy, which can be particularly ambiguous when the movement 
rates of the focal species are less than the spatial resolution of the GPS fixes [26]. Species-specific resampling strategies and correction factors can go some way to redressing this [cf. 26, 27, 28].

Many factors affect GPS performance including habitat type and heterogeneity [29-32], topography of the terrain [33,34], view of the sky availability [35], weather conditions [30], submersion in water [36, 37], time of day [38], vegetation cover/type [33,39], GPS orientation [40], fix acquisition rate [41, 42], in addition to the number of available satellites and their orbiting geometry with respect to one another $[43,44]$. All these elements affect the propagation of signal quality and/or receiver reception capability and thus increase triangulation error (see Hofman et al. [4] for review), with the latter two factors often assessed via the dilution of precision (DOP) values $[44,45]$.

Species-specific movements can be misinterpreted because GPS error often exaggerates the extent of movement, with error associated with distance measures being additive over time, and particularly germane at higher sampling frequencies (given that higher rates of error are incorporated per unit time) $[42,46]$. Indeed, although a number of authors have attempted to resolve the accuracy of GPS performance by quantifying the fix success rate and location error over various scenarios [cf. 47, 48], the critical modulator of GPS performance is animal behaviour [cf. 40, 49-52]. For example, Heard et al. [38] demonstrated that fix success rate for GPSs on grizzly bears (Ursus arctos) followed a bimodal circadian pattern, which was paralleled to the activity time-budgets of the bear, with higher forest density cover and variability in collar orientation being attributed to declines in fix rate. Similarly, after collaring both Eurasian lynx (Lynx lynx) and wolverine (Gulo gulo) in similar habitat, Mattisson et al. [50] suggested that high discrepancy in fix rate between the two species could be explained by differences in their behavioural repertoire. In essence, the specifics of animal movement, the 'what', 'where', 'when' and 'how' [cf. 1], underpins the species interaction with its environment and consequently the dual proficiency of signal propagation and reception between satellites and receiver. Resting is the most common behaviour for most terrestrial animals (particularly carnivores) and critically affects fix accuracy, because resting is typically associated with a change of body position (e.g., resting on the collar) and/or coverage within/near 'signal obstructing' environmental features (e.g., sleeping under trees or in caves/ burrows), thus decreasing the available sky for the GPS receiver [31-33,44]. This issue is compounded for collar-mounted GPS devices, because behaviours variously affect the position of the GPS antenna even though many collars are designed to be bottom-weighted to minimize this problem $[33,49,53]$.

Despite the well-documented issues of locational error and numerous mitigation strategies being proposed [54-57], there has been no 'gold standard' solution to identify inaccurate fixes. For example, Lewis et al. [43] emphasised using DOP values, removing fixes with values $>5$ and only keeping positions where $\geq 3$ satellites were registered to eliminate potentially large location errors. This recommendation was based on the premise that a wider geometry of satellite spacing, results in lower recorded DOP values and this along with a higher number of registered satellites, is associated with minimising triangulation error. The relationship between spatial precision and increasing DOP values, whilst generally accepted, is noisy however, and this approach can reduce datasets considerably, whilst 
still leaving notably anomalous fixes intact [34, 58]. Juxtaposed to this, Bjørneraas et al. [47] developed a method that focused on the movement characteristics of the focal species to identify large locational errors with minimal data reduction. This included screening for unrealistic distances travelled, speeds and turn angles between successive locations. However, this can become complicated and arbitrary at high sampling frequencies and is computationally intensive for large data sets.

To our knowledge, a specific solution for screening inaccurate locations from high resolution GPS data (e.g., $\geq 1 \mathrm{~Hz}$ ) has not yet been proposed. The difficulty is that, whilst shorter fix intervals are typically associated with higher fix accuracy $[41,49,59]$, locational error is, within the wider context of daily movement, relatively small and so harder to identify accurately. Disentangling this error is particularly relevant because GPS units with high fix rates used on animals are deployed with fine-scale analysis of movement trends in mind [cf. 26].

We note that since GPS 'jitter' (a term we use to define fixes inaccurately fluctuating around a central location) is disproportionately high during stationary periods $[37,41,46]$, the viability of deriving accurate movement from high resolution GPS trajectories depends on the ability to determine when an animal is moving or not in a manner that is independent of the GPS-derived movement. Studies have already used acceleration to activate GPS units only during movement, both as a means to increase battery longevity and avoid the fix inaccuracy prevalent during periods of inactivity [51, 60]. Properly coupled GPSacceleration systems are uncommon however, whilst a moving animal (as discerned from the accelerometer), does not necessarily correspond with a working GPS (e.g., due to signal obstruction, and because cold start 'blind' satellite searches are associated with lower fix success rates [41]). For highly resolved animal tracks, we advocate the importance of recording fixes continuously, in part to mitigate performance issues associated with cold starts between fix interval $[26,41,61]$ and also because finescale GPS estimates can be compared alongside acceleration data to aid in differentiating between nontravelling movements from travelling movements [cf. 62]. Beyond this, identifying 'hotspots' of GPS jitter may be useful for discerning GPS performance according to habitat type and/or behaviour. As part of this, we propose a new method for screening raw, high-resolution GPS data by accounting for the amount of activity using accelerometers and equating their outputs with an estimate of speed to evaluate the likelihood of movement per unit time. This is based on the observation that dynamic body acceleration (DBA - for definition see Wilson et al. [63]) increases approximately linearly with speed in terrestrial animals [64-66]. Thus, any GPS-derived speed should co-vary with DBA.

Here, we propose a decision tree-based framework in which user-defined thresholds of i) GPS speed, ii) DBA and iii) time, are implemented to screen GPS fixes and remove those that do not equate to genuine travelling movement. We also suggest an initial method for screening extreme anomalous fixes using distance estimates between the raw GPS track and the median filtered equivalent. We illustrate this using data from collar-fitted free-ranging lions (Panthera leo) within the Kgalagadi Transfrontier Park in the Kalahari Desert. The aims of this study are to provide both the conceptual and methodological protocol for screening high resolution GPS data using a Movement Verified Filtering (MVF) protocol and to discuss the broader applicability this method has for discerning animal movement. 


\section{Methods}

The procedure relates to 14 days of data derived from 11 wild lions ( 5 males and 6 females) in the Kgalagadi Transfrontier Park, South Africa, during February-March 2019. Lions were equipped with a LiteTrack GPS collar (Lotek Wireless Inc. [67]), to which a Gypsy_5 Techno-smart GPS unit (Technosmart s.r.l. [68]) set to record at $1 \mathrm{~Hz}$ and a 'Daily Diary' (DD) [containing inter alia tri-axial accelerometers and tri-axial magnetometers] [cf. 69] recording at $40 \mathrm{~Hz}$, were attached. The GPS units were encased in a thick 3D-printed Acrylonitrile butadiene styrene (ABS) plastic oval housing and DD's were enclosed in a watertight aluminium housing (cf. supplementary information (SI): Fig.1).

There were two collar sizes; small collars weighed $1.24 \mathrm{~kg}$ and large collars weighed $1.33 \mathrm{~kg}$ (attached with all devices), which constituted $<2 \%$ and $<1 \%$ of the body mass of the lightest equipped female and male animals, respectively. Data were retrieved from the collars approximately two weeks after the tag deployment. Intermittent behavioural observations of each pride took place at dawn and dusk, and occasionally during the day and night, for approximately 2-3 hours. During these periods, ethograms of the collared individual's various activities were recorded to document movement for comparison with the acceleration and GPS speed estimates to verify the accuracy of our MVF thresholds (Table 1). These observations were also carried to check for any potential negative side effects of the collars -none were apparent. See SI.1 for more information on the study site, capture protocol and devices used). All analyses were performed in Daily Diary Multi Trace (DDMT) [70], RStudio (open-source statistical programming software, [71]) and Origin pro 2016 (OriginLab Corporation, [72]).

\subsection{The Movement Verified Filtering (MVF) method}

The MVF protocol (illustrated in Fig. 1) primarily involves deriving DBA from tri-axial accelerometery data, computing speed from GPS data, and evaluating how both scale with each other during traveling movement. Specifically, the step-by-step method (used for lions) involves:

\section{Derivation of DBA}

Vectorial dynamic body acceleration (VeDBA) [73] was the DBA metric used for activity [63] and as a proxy for speed [64]. VeDBA is the vectorial sum of the dynamic body acceleration in a tri-axial acceleration signal (cf. SI. 2). A rolling mean was applied to raw VeDBA values (a 2 s centre-aligned window was used for lions) to ensure that both acceleration and deceleration components of an animal's stride cycle were incorporated together within any particular time period [63].

\section{Derivation of GPS speed}

The trigonometric Haversine formula $[74,75]$ was used to calculate the shortest distance between fixes of an appropriate stepping range (cf. SI. 2). We define a stepping range as the interval between each retained fix - a 5 -fix stepping range was used for lions (distance computed between every $5^{\text {th }}$ fix). Each successive distance estimate was divided by its time period (between retained fixes) to convert to GPS 
speed $(\mathrm{m} / \mathrm{s})$. A rolling mean was applied to GPS speed, (5 s centre-aligned rolling mean used for lions) for greater interpolation purposes with respect to acceleration estimates (cf. discussion and SI. 2, detailing the importance of a suitable stepping/post-smoothing range). Missing fixes were not included in the computation of GPS speed.

\section{Time synchronising GPS speed and DBA data}

Both VeDBA and GPS speed data were time-synchronised and sub-sampled to $1 \mathrm{~Hz}$ to make the data more manageable for analysis and because differentiating between fine-scale behaviours was not a prime objective of this study. Missing locational data were expressed as 'NA'.

\section{Using GPS-derived distance to identify extreme outliers - Distance threshold (Z)}

Missing locational data were replaced with values of the subsequent registered fix (RF). To identify extreme outliers, a median rolling filter was applied to both the longitude and latitude coordinates of the RF (MeFF). The Haversine method was then used to calculate the distance (units in metres) between the two sets of coordinates (RF vs MeFF) per unit time. Locational data (RF) above the Z threshold were deemed outliers (and thus failed the first step of the MVF protocol). By applying a rolling median using a suitable window length, large distance estimates reflecting either a single or multiple 'batched' outlier(s) could be distinguished from fixes deemed 'accurate' but highly separated in space due to large gaps in locational data. The window length size and $Z$ threshold should be chosen according to the animal in question due to the scales of movement undertaken by different species (median filter window length of $60 \mathrm{~s}$ and a lenient threshold of $100 \mathrm{~m}$ used for lions). The window length should be large enough so that the calculated median is not affected by a potential batch of consecutive anomalies at any one time. When plotted against time, the distance between RF vs MeFF shows relatively consistent variation about a given range (dependent on the window size set), though large obvious spikes indicate outliers, and the extent of this disparity can give an indication of the $Z$ threshold to set.

\section{Movement thresholds $(X \& Y)$}

The second stage for screening the GPS data were the thresholds of VeDBA $\left(\mathrm{X}_{\mathrm{VeDBA}}\right)$ and GPS speed $\left(\mathrm{Y}_{\mathrm{GPS}}\right)$ that infer moving behaviour. We set the protocol for fixes to fail the MVF protocol when;

(i) VeDBA $<$ X \& GPS speed $>$ Y (likely resultant from locational error)

(ii) VeDBA $>\mathrm{X} \&$ GPS speed $<\mathrm{Y}$ (likely resultant from a stationary behaviour)

Where $\mathrm{X}$ and $\mathrm{Y}$ were given defined thresholds.

For the lions, after initial inspection of data with respect to ground-truthed behavioural observations, the threshold $X$ was determined as $0.11 \mathrm{~g}$ and the threshold $Y$ was determined as $0.35 \mathrm{~m} / \mathrm{s}$ (see below). These thresholds were lenient, incorporating even slow movement and accounting for discrepancies of the relative magnitude of acceleration estimates between individuals [cf. 63, 76]. 


\section{Time threshold (T)}

The final stage of validating movement was to implement a minimum time threshold (T), over which uninterrupted movement had to occur before it was classified as such. This was implemented to discern travelling movement (where the animal location changed) from non-travelling movement (e.g., when the animal rolled over) for periods when both $\mathrm{X}_{\mathrm{VeDBA}}$ and $\mathrm{Y}_{\mathrm{GPS}}$ thresholds were met. MVF values were assigned a value of one, for every GPS fix that was time-matched to periods where the above thresholds $\left(X_{V e D B A}\right.$ and $\left.Y_{G P S}\right)$ were met for a minimum duration of $T$ ( $5 \mathrm{~s}$ was used for lions in the current study). MVF periods encoded as 1, occurring $\leq 2 \mathrm{~s}$ from one another were merged). An MVF value of zero represented either missing locational data, extreme outliers (identified by $Z$ threshold) or periods when the data indicated the animal was non-moving.

\subsection{Data analyses}

Various movement-derived metrics were compared between periods when animals were deemed to be moving ('travelling' movement) (MVF = 1) and periods when they were deemed to be non-moving ('nontravelling'/stationary movement) (MVF = 0). Such metrics include estimates of pitch, roll, heading, distance travelled, speed and tortuosity estimates (see SI. 4 for procedures and references therein). Here, unless otherwise stated, data ascribed as non-moving do not include data when GPS positions were missing or were extreme outliers (the latter, determined by the $z$ threshold as described above). Results presented as percentages are given as ' $x$ ' with variance as one standard deviation (SD) and range in the format; $\left[ \pm 1 \mathrm{SD}\left(\right.\right.$ range $_{\min }$ - range $\left.\left._{\max }\right)\right]$.

\section{Results}

Across 25 hours of behavioural observations, the MVF method using the thresholds outlined above registered an average accuracy of $97 \%$ (Table. 1; data correctly assigned as moving). This protocol was determined to have a high true negative rate (>99\%) and low false positive rate $(<1 \%)$, indicating that data that surpassed the MVF protocol indeed showed that the animal was moving with a high degree of certainty. True positive rate was slightly lower (c. $95 \%$ ) and was perceived to have been primarily modulated according to the variability in fix latency, which (irrespective of stepping/post-smoothing range) can result in a time delay, uncoupling estimates of GPS speed from the instantaneous and definitive expression of DBA estimates. It thus occasionally results in the beginning or end of periods that animals were moving being misclassified as 'non-moving' (MVF $=0)$. 
Table 1. Contingency table documenting the mean accuracy and misclassification rate of the MVF method from $~ 25$ hours of behavioural observations (ethograms) between eight individuals.

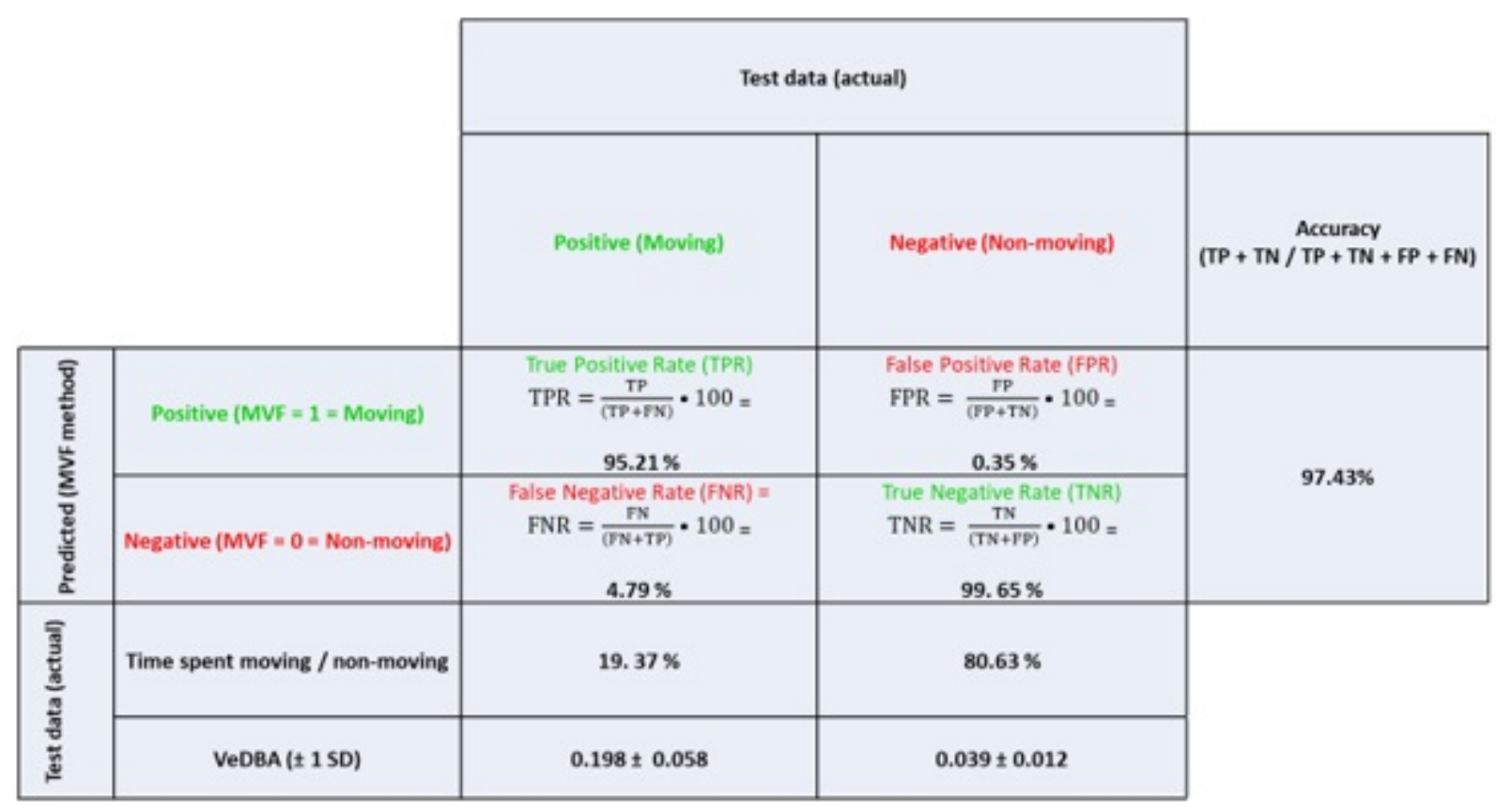

Fix success rate for the GPS varied between $89 \%$ and $97 \%$ across different animals. There was no indication of systematic drop-out (variability of fix success rate) being modulated according to time over the 14-day monitoring periods (SI. 3: Table S1 \& Fig. S1). Generally, GPS-derived speed correlated well with VeDBA $\left(r^{2}=0.74 \pm 0.04(0.67-0.81)\right)$ (SI. 2: Fig. S4), especially during periods that were defined by the MVF protocol as 'movement' (Fig. 3A \& C, SI. 2: Fig. S1:3). Discrepancies between GPS-speed and VeDBA were associated with location error (Fig. 2, SI. 2: Fig. S3), with the MVF approach highlighting that the position of the collar depended on the animal's behaviour (Fig. 4, SI. 3: Table S2) and that this was a prime modulator of GPS performance (cf. Fig. 2, Fig. 3B, SI. 2: Fig. S3).

On average, $13.3 \% \pm 3.3(8.3-19.5)$ of data acquisition passed the MVF protocol (SI. 3: Table 2). The majority of data deemed to be non-moving, was due to both $\mathrm{X}_{\mathrm{VEDBA}}$ and $\mathrm{Y}_{\mathrm{GPS}}$ thresholds not being met; $70.4 \% \pm 3.6(65-77)$. However, an appreciable proportion of non-moving data was due to the $Y_{G P S}$ threshold being met, but not the $\mathrm{X}_{\mathrm{VeDBA}}$ being met; $12.4 \% \pm 3.0(9-18)$ or both $\mathrm{Y}_{\mathrm{GPS}}$ and $\mathrm{X}_{\mathrm{VeDBA}}$ thresholds being met, but not for the duration of $\mathrm{T}_{\text {time }} ; 12.5 \% \pm 2.9(8-18)$. Data where $\mathrm{X}_{\mathrm{VeDBA}}$ was met, but not $\mathrm{Y}_{\mathrm{GPS}}$, comprised 4.85\% \pm 1.3 (3 - 7) (SI. 3: Fig. S2). The additive nature of errors associated with GPS jitter was significant and exemplified within cumulative distances moved (between fixes) (Fig. 5, SI. 3: Table S2) and apparent even at the broadest scales of movement (SI.2: Fig. S5). It was clear that GPS jitter was much more prominent when lions were resting and unless these data were filtered, use of these raw unfiltered GPS data resulted in biased and erroneous speed, distance and tortuosity of movement 
estimates (SI. 3: Table S2). Following the MVF method, there appeared to be a greater correlation between DD- and GPS-derived heading estimates (SI. 4: Fig. S1).

\section{Discussion}

\subsection{Evaluation of the MVF protocol}

This work demonstrates the value of using both DBA and GPS data to discern moving behaviours from stationary behaviours with a computationally quick protocol which effectively filters inaccurate fixes from high frequency GPS data (e.g., $\geq 1 \mathrm{~Hz}$, though possibly lower - cf. SI.2: Fig. S1 \& S2). The central premise is that when the magnitude of GPS speed and VeDBA both indicate movement (via pre-set thresholds), then movement is indeed likely (Table. 1, Fig. 2, Fig. 3A, SI.2: Fig S1 \& S3). This highlights the problem of GPS jitter when VeDBA does not correspond to movement even though the GPS indicates otherwise. Conversely, (relatively energetic) non-travelling behaviours are flagged up when the magnitude of VeDBA infers movement while data of GPS speed does not.

Results reaffirm the importance of screening GPS inaccuracies within high frequency independentlycollected datasets of animal movements, due to the additive nature of GPS jitter - most prevalent during rest periods (Fig. 2, SI. 3: Table S2 \& Fig. S3). This was particularly relevant in the current study because of the high proportion of data allocated to non-moving behaviours (SI.3: Table S2) (reflecting the energyconservation strategy that Kalahari lions adopt [cf. 77]). Indices of collar/postural offsets (evaluated using absolute values of pitch and roll) showed high variability during times when GPS units did not acquire fixes (Fig. 4), even when fix success rate could not be attributed to battery longevity (SI.3: Table S1, Fig. S1). Animal behaviour (including habitat selection) thus seems to be a primary factor affecting fix success rate and quality. Clear mono-modal peaks in the indices of posture were only witnessed when all thresholds of our MVF approach were met (Fig. 4). Whilst there were slight differences in the tightness of these distributions between lions (presumably due to discrepancies between collar fit), this does suggest that the optimum collar-body position for acquiring satellite signals occurred during travelling movement. In contrast, distributions were much more varied during times of non-moving, again highlighting the interplay between animal behaviour, collar orientation and GPS performance.

Our results highlight how, in the absence of appropriate filtering, inappropriate conclusions about a species' movement can be made. Here, there were stark contrasts of tortuosity, speed and most notably, distance travelled estimates between sets of data that both passed and failed our MVF method (SI. 3: Table S2). This method may therefore have particular value for distinguishing true small-scale arearestricted search (ARS) behaviour [78] by removing spurious turn angles caused by jitter [79, 80] (cf. SI.4: Fig. S1). Here, cumulative distance from non-moving data was $80 \%$ higher than their actual moving periods for some lions and this highly inflated index of movement was exemplified when measured as hourly averages (Fig. 5), apparently showing that lions travelled greater distances during the hottest parts of the day, something that is extremely unlikely [cf. 81]. Furthermore, our MVF protocol reduced the apparent maximum speed of any lion from $>150$ to $48 \mathrm{~km} / \mathrm{h}$. This critical issue highlights the drawbacks 
of assessing GPS data sampled at high frequency (in spite of necessary post-resampling strategies (SI.2)), which intensifies erroneous location estimates (cf. Fig. 2, SI.2: Fig. S3), even at macro-scales of movement (SI.2: Fig. S4).

\subsection{Utility of the MVF protocol according to species-specific and environmental circumstance}

The Haversine method for determination of animal speed and location using GPS positional fixes can estimate distances travelled with high precision, however for datasets containing many points collected at high frequencies, distance estimates are unreliable at small stepping ranges due to the interplay between location error and the precision of longitude and latitude coordinates that produce additive errors [26, 82]. Most commercial GPS units record fixes to five decimal places, with the fifth digit of the decimal place giving approximately $1.1 \mathrm{~m}$ resolution. Further, the computation time for a device to record a GPS fix can vary, reducing the synchronisation of time between both GPS and the accelerometer logger. Given that many terrestrial animals maintain relatively low travel speeds for extended periods [cf. 83], we note that appropriate choice of stepping range and smoothing window are critical for deducing reasonable step length estimates per unit time (SI.2: Fig. S1 \& S2), with this being dependent upon the (species-specific) scales of movement being assessed [cf. 64, 84].

Essentially, there is a trade-off between incorporating higher rates of error and increasing the lag of change relative to the properly time-synchronized acceleration data. This means that accurate fine-scale estimates of GPS-derived speed are not possible and so the relationship with body movement measurements such as VeDBA will never be succinct given the disparity of resolution from both measures. In addition, inter- and intra-specific variations of acceleration estimates can arise due to discrepancies of; morphology [64], locomotion mechanisms (e.g., change in gait to facilitate higher speeds [85]), extrinsic factors (e.g., moving over a deformable substrate / changeable grade [84, 86]), tag placement $[63]$ and collar roll $[87,88]$, thereby altering the relationship between VeDBA and mechanical power (and thus speed) $[84,89]$.

Alongside GPS resampling, MVF user-defined thresholds are expected to change according to the study species and scales of movement in question. For example, DBA estimates (specifically ODBA [63]) of African elephants (Loxodonta africana) typically ranged between 0.15 and $0.3 \mathrm{~g}$ during periods of walking [90] and this is comparable to that reported from Eurasian beavers (Castor fiber) $(0.265 \pm 0.029)$ [91], though notably, both species have different leg lengths and move with very different gaits which gives very different DBA-dependent speed estimates, as demonstrated by Bidder et al. [64] for multiple species.

It is notable here that we have focussed on terrestrial movement, and this is primarily because the relationship between DBA and speed can break down substantially for many aquatic and aerial species. This occurs because, for example, birds can glide at a variety of grounds speeds (depending on e.g., wind vectors and glide angle) without changing DBA, and air compression with depth affects the buoyancy of many marine animals, which complicates the DBA speed relationship depending on swim angle [92-94]. Furthermore, GPS is restricted to (potentially infrequent) resurfacing events for diving animals and so 
scaling DBA with GPS-derived speed is problematic for extended periods of time during underwater movements. Taken together, whilst we do not rule out extensions of the MVF method for use in such environments, we advocate that in its current form, it is most suitable for evaluating movements on land.

Importantly, the validity of this method is dependent on the interaction between a focal species' behaviour and where it inhabits - the critical limitation being the assumption that fixes are accurate during periods of moving. This is demonstrably not always the case (cf. Fig. 6), even in our study area, the Kgalagadi Transfrontier Park, which is open, with relatively sparse vegetation. Since vegetation type and density are key modulators of GPS accuracy $[35,38,39,43,48]$, the viability of our method needs to be tested within other (e.g., more vegetated) environments.

Nevertheless, for the study species in question, we have highlighted the effectiveness of this method and, in line with the above considerations, have demonstrated that a general correlation does exist between the magnitudes of both DBA and GPS speed during movement periods (Fig. 3, SI.2: Fig. S1:3). As such, we suggest that this approach could be used further to discern reliable events of high performance (e.g., hunt chases) and implemented within the dead-reckoning framework [cf. 65, 95], both as a corollary to the DBA-speed relationship (required for the speed coefficient) [65] and the GPS screening protocol prior to the correction process of dead-reckoned tracks [66]. At the very least, we demonstrate the utility for GPS speed to be included as a useful parameter for identifying behaviours and may be of value to more complex approaches (e.g., machine learning [cf. 96, 97-99], the lowest common denominator (LoCoD) method [100]) and space-state models [e.g., 101, 102] for precluding certain behaviours from movement and screening for location error. Indeed, applying this method as a validator of movement extent within behaviour-based studies over finely resolved space and time, may facilitate the powers of inference, such as when considering animal responses to human barriers [cf. 103]. Lastly, we theorise that high fix frequency will help elucidate fix inaccuracy within areas of high canopy cover, possibly via extensions to this method such as including upper threshold limits and comparing variation in GPS speed juxtaposed to DBA estimates and GPS- and DD-derived heading estimates (cf. SI. 4).

\section{Conclusion}

Here we reaffirm the importance of initial GPS screening so to avoid inaccurate movement estimates. Animal behaviour seems to be a major modulator of GPS performance and this is particularly germane in collared species due to the interaction between behaviour and collar orientation. The proposed Movement Verified Filtering method provides a basis for high-resolution GPS-screening, which is user-friendly, computationally quick and focuses on identifying behaviour to filter GPS data. Movement-defined thresholds can be modelled according to the focal species in question, whilst further differences between motion sensor and GPS derivatives can be incorporated into this MVF foundation to resolve fix inaccuracy during movement. Movement-based outputs comparing MVF values from lion data, exemplified the degree of inaccuracy associated with GPS jitter and the importance of removing such additive error prior to assessing fine-scale trends of movement, particularly step length. Our results show that consideration of data from both GPS units and motion sensors greatly helps validate true movement 
patterns and reaffirms the caution required when interpreting fine-scale GPS sampling such as during ARS analysis. Further work could assess the value of MVF for other species with different activities and habitat selections, particularly those that move within highly vegetated areas. The consequences of the errors introduced by GPS inaccuracies are broad, ranging from erroneous inferences of behaviour, movement, speed and energy budgets. The approach proposed here avoids these errors and enables accurate assessments of these traits.

\section{Declarations}

Ethics approval and consent to participate

Conditions and approvals were granted by the Animals Scientific Procedures Act (ASPA) at Queens University of Belfast (QUB-BS-AREC-18-006) and Pretoria University (NAS061-19), permit authorisation was given by South African National Parks (Permit Number SCAM 1550).

\section{Consent for publication}

Not applicable

Availability of data and materials

We provide a step-by step R script for implementing the MVF method on an example lion dataset (containing DD and GPS data files). The R script and example data set will be made available on GitHub [available at 104] if the manuscript is accepted for publication.

\section{Competing interests}

The authors declare that they have no competing interests

\section{Funding}

This research contributes to the CAASE project funded by King Abdullah University of Science and Technology (KAUST) under the KAUST Sensor Initiative. Fieldwork was supported in part by a Department for Economy Global Challenges Research Fund grant to MS.

\section{Author Contributions}

RMG conceived the study and RMG and RW wrote the initial draft. PH constructed tag housings. Data collection (capture of lions for fitting and removal of technology) was led by SF, DG, PV, LVS and AB, with assistance from MS, SB, MCVR, RMG, MB, CJT and PH. RMG conducted the analysis and all authors contributed to manuscript revision.

Acknowledgments 
We thank SANParks and the Department of Wildlife and National Parks, Botswana, for allowing our research in the Kgalagadi Transfrontier Park We are grateful for support and kind assistance of the staff and Rangers at the Kgalagadi National Park who were involved with this work, especially Steven Smith, Christa von Elling, Wayne Oppel and Corera Links.

\section{References}

1. Nathan R, Getz WM, Revilla E, Holyoak M, Kadmon R, Saltz D, Smouse PE: A movement ecology paradigm for unifying organismal movement research. Proceedings of the National Academy of Sciences 2008, 105(49):19052-19059.

2. Cagnacci F, Boitani L, Powell RA, Boyce MS: Animal ecology meets GPS-based radiotelemetry: a perfect storm of opportunities and challenges. Philosophical Transactions of the Royal Society B: Biological Sciences 2010, 365(1550):2157-2162.

3. Latham ADM, Latham MC, Anderson DP, Cruz J, Herries D, Hebblewhite M: The GPS craze: six questions to address before deciding to deploy GPS technology on wildlife. New Zealand Journal of Ecology 2015, 39(1):143-152.

4. Hofman MPG, Hayward MW, Heim M, Marchand P, Rolandsen CM, Mattisson J, Urbano F, Heurich M, Mysterud A, Melzheimer J, Morellet N, Voigt U, Allen BL, Gehr B, Rouco C, Ullmann W, Holand Ø, Jørgensen NH, Steinheim G, Cagnacci F, Kroeschel M, Kaczensky P, Buuveibaatar B, Payne JC, Palmegiani I, Jerina K, Kjellander P, Johansson Ö, LaPoint S, Bayrakcismith R, Linnell JDC, Zaccaroni M, Jorge MLS, Oshima JEF, Songhurst A, Fischer C, Mc Bride RT, Jr., Thompson JJ, Streif S, Sandfort R, Bonenfant C, Drouilly M, Klapproth M, Zinner D, Yarnell R, Stronza A, Wilmott L, Meisingset E, Thaker M, Vanak AT, Nicoloso S, Graeber R, Said S, Boudreau MR, Devlin A, Hoogesteijn R, May-Junior JA, Nifong JC, Odden J, Quigley HB, Tortato F, Parker DM, Caso A, Perrine J, Tellaeche C, Zieba F, Zwijacz-Kozica T, Appel CL, Axsom I, Bean WT, Cristescu B, Périquet S, Teichman KJ, Karpanty S, Licoppe A, Menges V, Black K, Scheppers TL, Schai-Braun SC, Azevedo FC, Lemos FG, Payne A, Swanepoel LH, Weckworth BV, Berger A, Bertassoni A, McCulloch G, Šustr P, Athreya V, Bockmuhl D, Casaer J, Ekori A, Melovski D, Richard-Hansen C, van de Vyver D, Reyna-Hurtado R, Robardet E, Selva N, Sergiel A, Farhadinia MS, Sunde P, Portas R, Ambarli H, Berzins R, Kappeler PM, Mann GK, Pyritz L, Bissett C, Grant T, Steinmetz R, Swedell L, Welch RJ, Armenteras D, Bidder OR, González TM, Rosenblatt A, Kachel S, Balkenhol N: Right on track? Performance of satellite telemetry in terrestrial wildlife research. PLOS ONE 2019, 14(5):e0216223.

5. Dore KM, Hansen MF, Klegarth AR, Fichtel C, Koch F, Springer A, Kappeler P, Parga JA, Humle T, Colin C, Raballand E, Huang Z-P, Qi X-G, Di Fiore A, Link A, Stevenson PR, Stark DJ, Tan N, Gallagher CA, Anderson CJ, Campbell CJ, Kenyon M, Pebsworth P, Sprague D, Jones-Engel L, Fuentes A: Review of GPS collar deployments and performance on nonhuman primates. Primates 2020, 61(3):1-15.

6. Pfeiffer T, Meyburg B-U: GPS tracking of Red Kites (Milvus milvus) reveals fledgling number is negatively correlated with home range size. Journal of Ornithology 2015, 156(4):963-975. 
7. Christiansen F, Esteban N, Mortimer JA, Dujon AM, Hays GC: Diel and seasonal patterns in activity and home range size of green turtles on their foraging grounds revealed by extended Fastloc-GPS tracking. Marine Biology 2016, 164(1):10.

8. Galanti V, Tosi G, Rossi R, Foley C: The use of GPS radio-collars to track elephants (<em>Loxodonta africana</em>) in the Tarangire National Park (Tanzania). Hystrix, the Italian Journal of Mammalogy 2000, 11(2).

9. García-Ripollés C, López-López P, Urios V: First description of migration and wintering of adult Egyptian Vultures Neophron percnopterus tracked by GPS satellite telemetry. Bird Study 2010, 57(2):261265.

10. Yamaç E, Bilgin CC: Post-fledging movements of Cinereous Vultures Aegypius monachus in Turkey revealed by GPS telemetry. Ardea 2012, 100(2):149-156.

11. Skarin A, Danell Ö, Bergström R, Moen J: Summer habitat preferences of GPS-collared reindeer Rangifer tarandus tarandus. Wildlife Biology 2008, 14(1):1-15.

12. Nielson RM, Manly BFJ, McDonald LL, Sawyer H, McDonald TL: Estimating habitat selection when GPS fix success is less than 100\%. Ecology 2009, 90(10):2956-2962.

13. Rumble MA, Benkobi L, Lindzey F, Gamo RS: Evaluating elk habitat interactions with GPS collars. In: Tracking animals with GPS: 12-13 March 2001 2001; Macaulay Land Use Research Institute, Aberdeen, UK: Macaulay Institute. 11-17.

14. McDuie F, Casazza ML, Overton CT, Herzog MP, Hartman CA, Peterson SH, Feldheim CL, Ackerman JT: GPS tracking data reveals daily spatio-temporal movement patterns of waterfowl. Movement Ecology 2019, 7(1):6.

15. Ungar ED, Henkin Z, Gutman M, Dolev A, Genizi A, Ganskopp D: Inference of animal activity from GPS collar data on free-ranging cattle. Rangeland Ecology \& Management 2005, 58(3):256-266.

16. Owen-Smith N, Goodall V: Coping with savanna seasonality: comparative daily activity patterns of African ungulates as revealed by GPS telemetry. Journal of Zoology 2014, 293(3):181-191.

17. Cristescu B, Stenhouse GB, Boyce MS: Predicting multiple behaviors from GPS radiocollar cluster data. Behavioral Ecology 2015, 26(2):452-464.

18. Hacker CE, Horback KM, Miller LJ: GPS technology as a proxy tool for determining relationships in social animals: An example with African elephants. Applied Animal Behaviour Science 2015, 163:175182.

19. Recio MR, Mathieu R, Latham MC, Latham ADM, Seddon PJ: Quantifying fine-scale resource selection by introduced European hedgehogs (Erinaceus europaeus) in ecologically sensitive areas. 
20. Liu D, Zhang G, Jiang H, Chen L, Meng D, Lu J: Seasonal dispersal and longitudinal migration in the Relict Gull Larus relictus across the Inner-Mongolian Plateau. PeerJ 2017, 5:e3380.

21. Hallworth MT, Marra PP: Miniaturized GPS Tags Identify Non-breeding Territories of a Small Breeding Migratory Songbird. Scientific Reports 2015, 5(1):11069.

22. Gibb R, Shoji A, Fayet AL, Perrins CM, Guilford T, Freeman R: Remotely sensed wind speed predicts soaring behaviour in a wide-ranging pelagic seabird. Journal of The Royal Society Interface 2017, 14(132):20170262.

23. Blecha KA, Alldredge MW: Improvements on GPS Location Cluster Analysis for the Prediction of Large Carnivore Feeding Activities: Ground-Truth Detection Probability and Inclusion of Activity Sensor Measures. PLOS ONE 2015, 10(9):e0138915.

24. de Weerd N, van Langevelde F, van Oeveren H, Nolet BA, Kölzsch A, Prins HHT, de Boer WF: Deriving Animal Behaviour from High-Frequency GPS: Tracking Cows in Open and Forested Habitat. PLOS ONE 2015, 10(6):e0129030.

25. Wang Y, Nickel B, Rutishauser M, Bryce CM, Williams TM, Elkaim G, Wilmers CC: Movement, resting, and attack behaviors of wild pumas are revealed by tri-axial accelerometer measurements. Movement ecology 2015, 3(1):1-12.

26. Ryan PG, Petersen SL, Peters G, Grémillet D: GPS tracking a marine predator: the effects of precision, resolution and sampling rate on foraging tracks of African Penguins. Marine Biology 2004, 145(2):215-223.

27. Humphries NE, Weimerskirch H, Sims DW: A new approach for objective identification of turns and steps in organism movement data relevant to random walk modelling. Methods in Ecology and Evolution 2013, 4(10):930-938.

28. Edelhoff H, Signer J, Balkenhol N: Path segmentation for beginners: an overview of current methods for detecting changes in animal movement patterns. Movement Ecology 2016, 4(1):21.

29. Janeau G, Adrados C, Joachim J, Gendner J-P, Pépin D: Performance of differential GPS collars in temperate mountain forest. Comptes Rendus Biologies 2004, 327(12):1143-1149.

30. Swanepoel LH, Dalerum F, Van Hoven W: Factors affecting location failure of GPS collars fitted to African leopards (Panthera pardus). African Journal of Wildlife Research 2010, 40(1):10-15.

31. Smith BJ, Hart KM, Mazzotti FJ, Basille M, Romagosa CM: Evaluating GPS biologging technology for studying spatial ecology of large constricting snakes. Animal Biotelemetry 2018, 6(1):1. 
32. Cochrane MM, Brown DJ, Moen RA: GPS Technology for Semi-Aquatic Turtle Research. Diversity 2019, 11(3):34.

33. Cain JW, Krausman PR, Jansen BD, Morgart JR: Influence of topography and GPS fix interval on GPS collar performance. Wildlife Society Bulletin 2005, 33(3):926-934.

34. Ironside KE, Mattson DJ, Arundel TR, Hansen JR: Is GPS telemetry location error screening beneficial? Wildlife Biology 2017, 2017(17).

35. Adams AL, Dickinson KJM, Robertson BC, van Heezik Y: An Evaluation of the Accuracy and Performance of Lightweight GPS Collars in a Suburban Environment. PLOS ONE 2013, 8(7):e68496.

36. Quaglietta L, Martins BH, de Jongh A, Mira A, Boitani L: A Low-Cost GPS GSM/GPRS Telemetry System: Performance in Stationary Field Tests and Preliminary Data on Wild Otters (Lutra lutra). PLOS ONE 2012, 7(1):e29235.

37. Justicia LS, Rosell F, Mayer M: Performance of GPS units for deployment on semiaquatic animals. PLOS ONE 2018, 13(12):e0207938.

38. Heard DC, Ciarniello LM, Seip DR: Grizzly Bear Behavior and Global Positioning System Collar Fix Rates. The Journal of Wildlife Management 2008, 72(3):596-602.

39. Hansen MC, Riggs RA: Accuracy, Precision, and Observation Rates of Global Positioning System Telemetry Collars. The Journal of Wildlife Management 2008, 72(2):518-526.

40. D'eon RG, Delparte D: Effects of radio-collar position and orientation on GPS radio-collar performance, and the implications of PDOP in data screening. Journal of Applied Ecology 2005, 42(2):383-388.

41. Forin-Wiart M-A, Hubert P, Sirguey P, Poulle M-L: Performance and Accuracy of Lightweight and Low-Cost GPS Data Loggers According to Antenna Positions, Fix Intervals, Habitats and Animal Movements. PLOS ONE 2015, 10(6):e0129271.

42. McGavin SL, Bishop-Hurley GJ, Charmley E, Greenwood PL, Callaghan MJ: Effect of GPS sample interval and paddock size on estimates of distance travelled by grazing cattle in rangeland, Australia. The Rangeland Journal 2018, 40(1):55-64.

43. Lewis JS, Rachlow JL, Garton EO, Vierling LA: Effects of habitat on GPS collar performance: using data screening to reduce location error. Journal of Applied Ecology 2007, 44(3):663-671.

44. Vance JA, Jachowski DS, Boynton AC, Kelly MJ: Importance of evaluating GPS telemetry collar performance in monitoring reintroduced populations. Wildlife Society Bulletin 2017, 41(4):729-735. 
45. Dussault C, Courtois R, Ouellet J-P, Huot J: Influence of Satellite Geometry and Differential Correction on GPS Location Accuracy. Wildlife Society Bulletin (1973-2006) 2001, 29(1):171-179.

46. Ganskopp DC, Johnson DD: GPS Error in Studies Addressing Animal Movements and Activities. Rangeland Ecology \& Management 2007, 60(4):350-358.

47. Bjørneraas K, Van Moorter B, Rolandsen CM, Herfindal I: Screening Global Positioning System Location Data for Errors Using Animal Movement Characteristics. The Journal of Wildlife Management 2010, 74(6):1361-1366.

48. Frair JL, Fieberg J, Hebblewhite M, Cagnacci F, DeCesare NJ, Pedrotti L: Resolving issues of imprecise and habitat-biased locations in ecological analyses using GPS telemetry data. Philosophical Transactions of the Royal Society B: Biological Sciences 2010, 365(1550):2187-2200.

49. Jiang Z, Sugita M, Kitahara M, Takatsuki S, Goto T, Yoshida Y: Effects of habitat feature, antenna position, movement, and fix interval on GPS radio collar performance in Mount Fuji, central Japan. Ecological Research 2008, 23(3):581-588.

50. Mattisson J, Andrén H, Persson J, Segerström P: Effects of Species Behavior on Global Positioning System Collar Fix Rates. The Journal of Wildlife Management 2010, 74(3):557-563.

51. Brown DD, LaPoint S, Kays R, Heidrich W, Kümmeth F, Wikelski M: Accelerometer-informed GPS telemetry: Reducing the trade-off between resolution and longevity. Wildlife Society Bulletin 2012, 36(1):139-146.

52. Jung TS, Kuba K: Performance of GPS collars on free-ranging bison (Bison bison) in northwestern Canada. Wildlife Research 2015, 42(4):315-323.

53. Belant JL: Effects of Antenna Orientation and Vegetation on Global Positioning System Telemetry Collar Performance. Northeastern Naturalist 2009, 16(4):577-584, 578.

54. Frair JL, Nielsen SE, Merrill EH, Lele SR, Boyce MS, Munro RHM, Stenhouse GB, Beyer HL: Removing GPS collar bias in habitat selection studies. Journal of Applied Ecology 2004, 41(2):201-212.

55. Visscher DR: GPS measurement error and resource selection functions in a fragmented landscape. Ecography 2006, 29(3):458-464.

56. Patel A, Stocks B, Fisher C, Nicolls F, Boje E: Tracking the Cheetah Tail Using Animal-Borne Cameras, GPS, and an IMU. IEEE Sensors Letters 2017, 1(4):1-4.

57. Joo R, Boone ME, Clay TA, Patrick SC, Clusella-Trullas S, Basille M: Navigating through the $\mathbf{R}$ packages for movement. Journal of Animal Ecology 2020, 89(1):248-267. 
58. Buerkert A, Schlecht E: Performance of three GPS collars to monitor goats' grazing itineraries on mountain pastures. computers and electronics in agriculture 2009, 65(1):85-92.

59. McGregor HW, Legge SM, Jones ME, Johnson CN: GPS collars are more efficient when collecting high-frequency data. Australian Mammalogy 2016, 38(2):237-240.

60. Wilson AM, Lowe J, Roskilly K, Hudson PE, Golabek K, McNutt J: Locomotion dynamics of hunting in wild cheetahs. Nature 2013, 498(7453):185-189.

61. Moriarty KM, Epps CW: Retained satellite information influences performance of GPS devices in a forested ecosystem. Wildlife Society Bulletin 2015, 39(2):349-357.

62. Watanabe S, Izawa $M$, Kato A, Ropert-Coudert $Y$, Naito $Y$ : A new technique for monitoring the detailed behaviour of terrestrial animals: A case study with the domestic cat. Applied Animal Behaviour Science 2005, 94(1):117-131.

63. Wilson RP, Börger L, Holton MD, Scantlebury DM, Gómez-Laich A, Quintana F, Rosell F, Graf PM, Williams H, Gunner R, Hopkins L, Marks N, Geraldi NR, Duarte CM, Scott R, Strano MS, Robotka H, Eizaguirre C, Fahlman A, Shepard ELC: Estimates for energy expenditure in free-living animals using acceleration proxies: A reappraisal. Journal of Animal Ecology 2020, 89(1):161-172.

64. Bidder OR, Soresina M, Shepard ELC, Halsey LG, Quintana F, Gómez-Laich A, Wilson RP: The need for speed: testing acceleration for estimating animal travel rates in terrestrial dead-reckoning systems. Zoology 2012, 115(1):58-64.

65. Bidder OR, Walker JS, Jones MW, Holton MD, Urge P, Scantlebury DM, Marks NJ, Magowan EA, Maguire IE, Wilson RP: Step by step: reconstruction of terrestrial animal movement paths by deadreckoning. Movement Ecology 2015, 3(1):23.

66. Dewhirst OP, Evans HK, Roskilly K, Harvey RJ, Hubel TY, Wilson AM: Improving the accuracy of estimates of animal path and travel distance using GPS drift-corrected dead reckoning. Ecology and Evolution 2016, 6(17):6210-6222.

67. Lotek products. Available at https://www.lotek.com/. Accessed 07 March 2021

68. Technosmart GPS tracking systems for animals. Available at https://www.technosmart.eu/. Accessed 07 March 2021.

69. Wilson RP, Shepard E, Liebsch N: Prying into the intimate details of animal lives: use of a daily diary on animals. Endangered Species Research 2008, 4(1-2):123-137.

70. Wildbyte Technologies. Available at http://www.wildbytetechnologies.com/. Accessed 07 March 2021. 
71. The R Project for Statistical Computing. Available at https://www.r-project.org/. Accessed 07 March 2021.

72. Origin Pro Graphing \& Analysis. Available a https://www.originlab.com/origin. Accessed 04 June 2021.

73. Qasem L, Cardew A, Wilson A, Griffiths I, Halsey LG, Shepard ELC, Gleiss AC, Wilson R: Tri-Axial Dynamic Acceleration as a Proxy for Animal Energy Expenditure; Should We Be Summing Values or Calculating the Vector? PLOS ONE 2012, 7(2):e31187.

74. Chopde NR, Nichat MK: Landmark based shortest path detection by using $A^{*}$ and Haversine formula. International Journal of Innovative Research in Computer and Communication Engineering 2013, 1(2):298-302.

75. Harja YD, Sarno R: Determine the best option for nearest medical services using Google maps API, Haversine and TOPSIS algorithm. In: 2018 International Conference on Information and Communications Technology (ICOIACT): 6-7 March 2018 2018. 814-819.

76. Williams HJ, Holton MD, Shepard ELC, Largey N, Norman B, Ryan PG, Duriez O, Scantlebury M, Quintana F, Magowan EA, Marks NJ, Alagaili AN, Bennett NC, Wilson RP: Identification of animal movement patterns using tri-axial magnetometry. Movement Ecology 2017, 5(1):6.

77. Hill RW, Wyse GA, Anderson M, Anderson M: Animal physiology, vol. 2. Sunderland, MA: Sinauer Associates 2004.

78. Weimerskirch H, Pinaud D, Pawlowski F, Bost CA: Does Prey Capture Induce Area-Restricted Search? A Fine-Scale Study Using GPS in a Marine Predator, the Wandering Albatross. The American Naturalist 2007, 170(5):734-743.

79. Hurford A: GPS Measurement Error Gives Rise to Spurious $180^{\circ}$ Turning Angles and Strong Directional Biases in Animal Movement Data. PLOS ONE 2009, 4(5):e5632.

80. DeCesare NJ, Squires JR, Kolbe JA: Effect of forest canopy on GPS-based movement data. Wildlife Society Bulletin 2005, 33(3):935-941.

81. Packer C, Swanson A, Ikanda D, Kushnir H: Fear of darkness, the full moon and the nocturnal ecology of African lions. PLOS ONE 2011, 6(7):e22285.

82. Laube P, Purves RS: How fast is a cow? Cross-Scale Analysis of Movement Data. Transactions in GIS 2011, 15(3):401-418.

83. Perry AK, Blickhan R, Biewener AA, Heglund NC, Taylor CR: Preferred speeds in terrestrial vertebrates: are they equivalent? Journal of Experimental Biology 1988, 137(1):207-219. 
84. Bidder OR, Qasem LA, Wilson RP: On Higher Ground: How Well Can Dynamic Body Acceleration Determine Speed in Variable Terrain? PLOS ONE 2012, 7(11):e50556.

85. Halsey LG, Shepard ELC, Hulston CJ, Venables MC, White CR, Jeukendrup AE, Wilson RP: Acceleration versus heart rate for estimating energy expenditure and speed during locomotion in animals: Tests with an easy model species, Homo sapiens. Zoology 2008, 111(3):231-241.

86. Kerdok AE, Biewener AA, McMahon TA, Weyand PG, Herr HM: Energetics and mechanics of human running on surfaces of different stiffnesses. Journal of Applied Physiology 2002, 92(2):469-478.

87. Silvy NJ, Lopez RR, Peterson MJ: Wildlife marking techniques. The Wildlife Society: Bethesda, MD; 2005.

88. Shepard EL, Wilson RP, Halsey LG, Quintana F, Laich AG, Gleiss AC, Liebsch N, Myers AE, Norman B: Derivation of body motion via appropriate smoothing of acceleration data. Aquatic Biology 2008, 4(3):235-241.

89. Gleiss AC, Wilson RP, Shepard ELC: Making overall dynamic body acceleration work: on the theory of acceleration as a proxy for energy expenditure. Methods in Ecology and Evolution 2011, 2(1):2333.

90. Soltis J, King L, Vollrath F, Douglas-Hamilton I: Accelerometers and simple algorithms identify activity budgets and body orientation in African elephants Loxodonta africana. Endangered Species Research 2016, 31:1-12.

91. Graf PM, Wilson RP, Qasem L, Hackländer K, Rosell F: The Use of Acceleration to Code for Animal Behaviours; A Case Study in Free-Ranging Eurasian Beavers Castor fiber. PLOS ONE 2015, 10(8):e0136751.

92. Wilson RP, Hustler K, Ryan PG, Burger AE, Noldeke EC: Diving birds in cold water: do Archimedes and Boyle determine energetic costs? The American Naturalist 1992, 140(2):179-200.

93. Williams $\mathrm{H}$, Shepard E, Duriez O, Lambertucci SA: Can accelerometry be used to distinguish between flight types in soaring birds? Animal Biotelemetry 2015, 3(1):1-11.

94. Gunner RM, Holton MD, Scantlebury DM, Van Schalkwyk OL, English HM, Williams HJ, Hopkins P, Quintana F, Gómez-Laich A, Börger L, Redcliffe J, Yoda K, Yamamoto T, Ferreira S, Govender D, Viljoen P, Bruns A, Bell SH, Marks NJ, Bennett NC, Tonini MH, Duarte CM, Van Rooyen MC, Bertelsen MF, Tambling $\mathrm{CJ}$, Wilson RP: Dead-reckoning animal movements in $\mathrm{R}-\mathrm{A}$ reappraisal using Gundog.Tracks. Animal Biotelemetry (in press).

95. Walker JS, Jones MW, Laramee RS, Holton MD, Shepard ELC, Williams HJ, Scantlebury DM, Marks NJ, Magowan EA, Maguire IE, Bidder OR, Di Virgilio A, Wilson RP: Prying into the intimate secrets of 
animal lives; software beyond hardware for comprehensive annotation in 'Daily Diary' tags. Movement Ecology 2015, 3(1):29.

96. Bidder OR, Campbell HA, Gómez-Laich A, Urgé P, Walker J, Cai Y, Gao L, Quintana F, Wilson RP: Love Thy Neighbour: Automatic Animal Behavioural Classification of Acceleration Data Using the KNearest Neighbour Algorithm. PLOS ONE 2014, 9(2):e88609.

97. Samarasinghe S: Neural Networks for Applied Sciences and Engineering: From Fundamentals to Complex Pattern Recognition: CRC Press; 2016.

98. Fehlmann G, O'Riain MJ, Hopkins PW, O'Sullivan J, Holton MD, Shepard ELC, King AJ: Identification of behaviours from accelerometer data in a wild social primate. Animal Biotelemetry 2017, 5(1):6.

99. Goodall VL, Ferreira SM, Funston PJ, Maruping-Mzileni N: Uncovering hidden states in African lion movement data using hidden Markov models. Wildlife Research 2019, 46(4):296-303.

100. Wilson RP, Holton MD, di Virgilio A, Williams H, Shepard ELC, Lambertucci S, Quintana F, Sala JE, Balaji B, Lee ES, Srivastava M, Scantlebury DM, Duarte CM: Give the machine a hand: A Boolean timebased decision-tree template for rapidly finding animal behaviours in multisensor data. Methods in Ecology and Evolution 2018, 9(11):2206-2215.

101. Fleming CH, Drescher-Lehman J, Noonan MJ, Akre TSB, Brown DJ, Cochrane MM, Dejid N, DeNicola V, DePerno CS, Dunlop JN, Gould NP, Hollins J, Ishii H, Kaneko Y, Kays R, Killen SS, Koeck B, Lambertucci SA, LaPoint SD, Medici EP, Meyburg B-U, Miller TA, Moen RA, Mueller T, Pfeiffer T, Pike KN, Roulin A, Safi K, Séchaud R, Scharf AK, Shephard JM, Stabach JA, Stein K, Tonra CM, Yamazaki K, Fagan WF, Calabrese JM: A comprehensive framework for handling location error in animal tracking data*. bioRxiv 2020:2020.2006.2012.130195.

102. Jonsen ID, Patterson TA, Costa DP, Doherty PD, Godley BJ, Grecian WJ, Guinet C, Hoenner X, Kienle SS, Robinson PW, Votier SC, Whiting S, Witt MJ, Hindell MA, Harcourt RG, McMahon CR: A continuous-time state-space model for rapid quality control of argos locations from animal-borne tags. Movement Ecology 2020, 8(1):31.

103. Jacobson SL, Bliss-Ketchum LL, de Rivera CE, Smith WP: A behavior-based framework for assessing barrier effects to wildlife from vehicle traffic volume. Ecosphere 2016, 7(4):e01345.

104. Gundog.Tracks GitHub database. Available at https://github.com/Richard6195/Dead-reckoninganimal-movements-in-R. Accessed 29 May 2021

Figures 


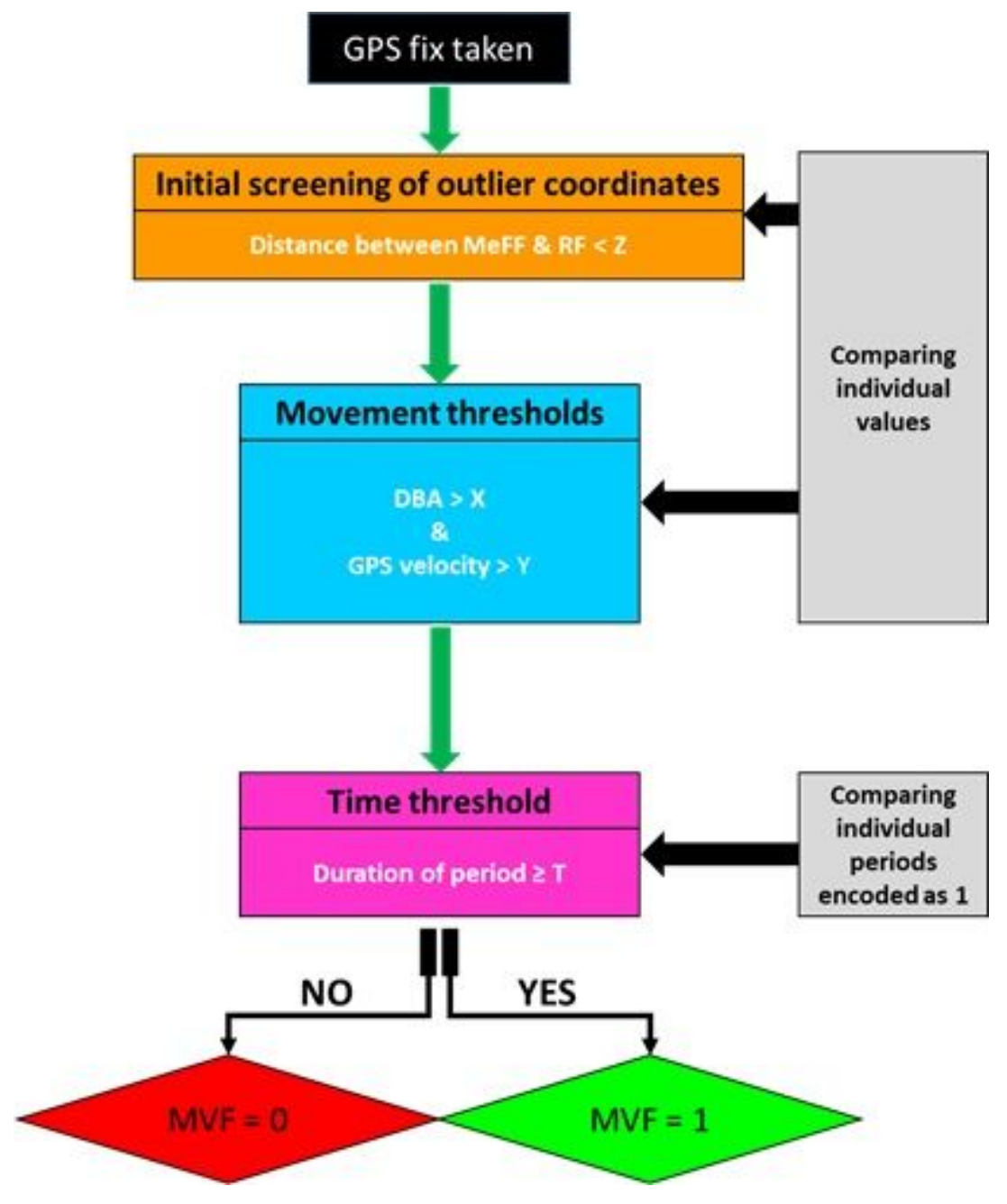

Figure 1

Schematic of the derivation of movement verified filtering (MVF). GPS fixes with an MVF value of 1 are considered to be more accurate given the data indicates travelling. Note values used at each stage (including the stepping range and post-smoothing windows in the prior derivations of GPS speed and VeDBA) are user-defined and must be adapted for the study 


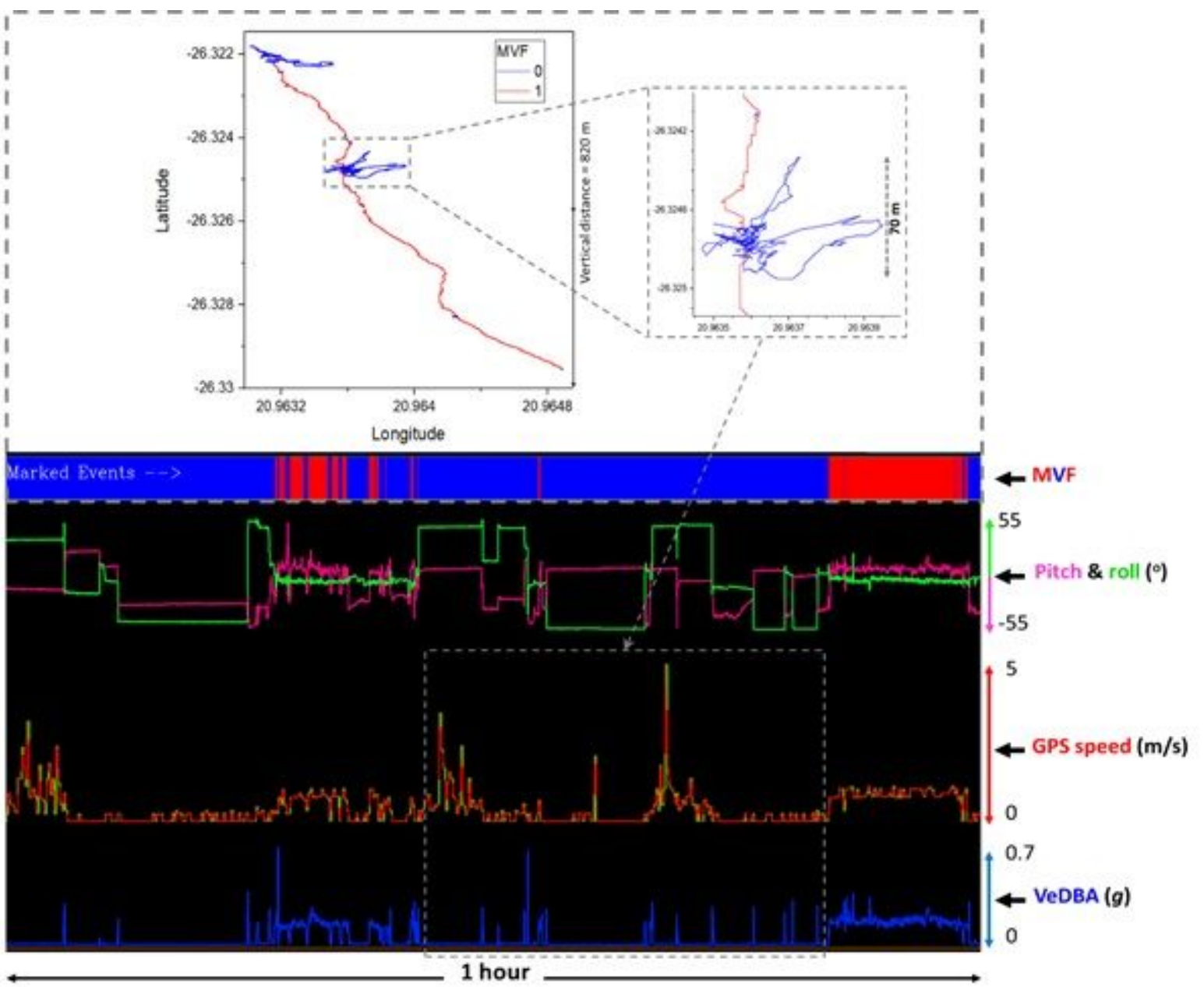

Figure 2

DD- and GPS-derived data showing intermittent periods of moving and stationary behaviours (lower panel). Note how many of the periods determined as non-moving (MVF $=0=$ blue) had high estimates of GPS speed (green = unsmoothed) due to large locational errors and this often followed sharp peaks in VeDBA, coinciding with a postural change (non-travelling behaviour). Note also how closely GPS speed estimates follow the VeDBA trace during periods of predominantly moving (MVF $=1=$ red) and the consistency of pitch and roll values (with intermittent bouts of stationary behaviour associated with a change in collar angle. The GPS fixes (upper panel) are coloured according to MVF values and exemplify high vertical straight-line distance between track coordinates due to GPS jitter. 


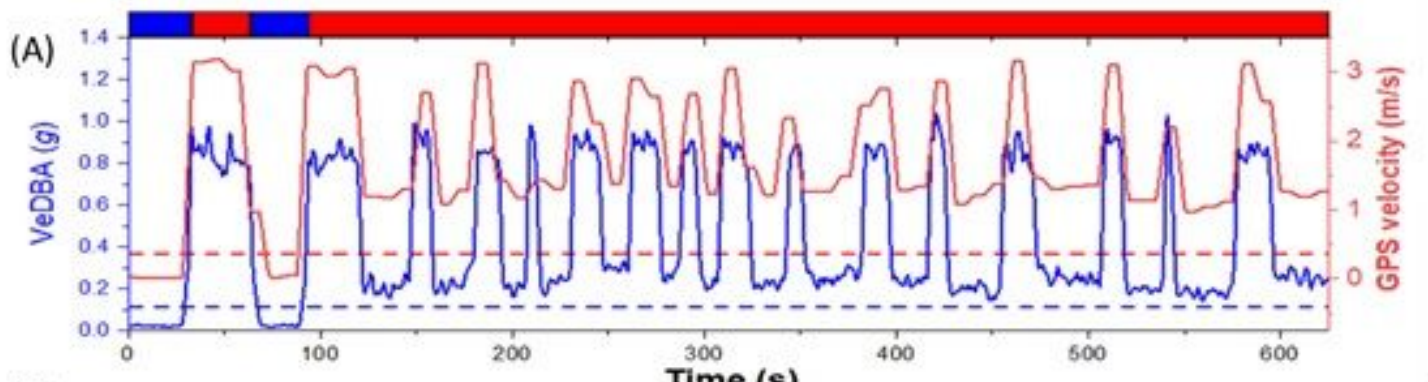

(B)
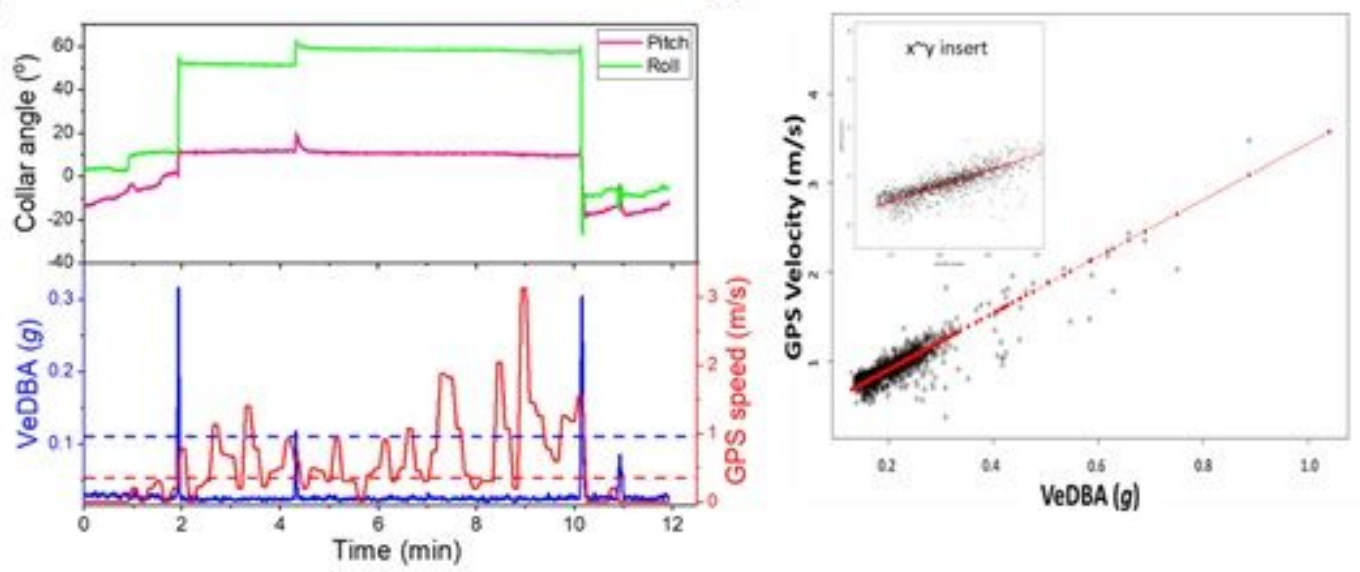

(C)

\section{Figure 3}

Example of the movement-based thresholds. (A) A period of predominately continuous movement (coloured rug at the top of plot denotes MVF values ( $1=$ moving (red), 0 =non-moving (blue)). The peaks of both VeDBA and GPS velocity are due to bouts of running, interspaced by either non-moving or walking bouts. (B) Relationship between VeDBA and GPS speed during a rest period, whereby the individual carried out a transitionary roll while lying prone (at approx. the 2-minute mark; as depicted by the pitch and roll angles), after which, GPS jitter became more apparent (as demonstrated by the higher variance in GPS speed estimates). (C) GPS speed $\sim$ VeDBA relationship for a given lion with linear regression $(y=a+$ bx and zoomed in the inset). Data from (C) are taken only from marked moving periods following the MVF method. Each data point represents the mean value per period, taken from ca. two weeks of data acquisition. 
(A)
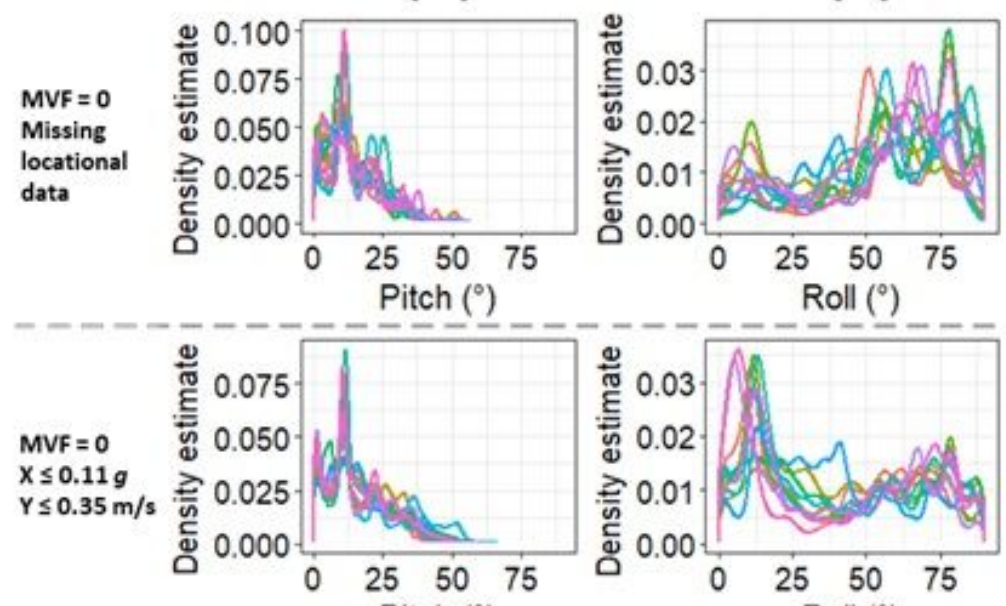

Roll $\left({ }^{\circ}\right)$

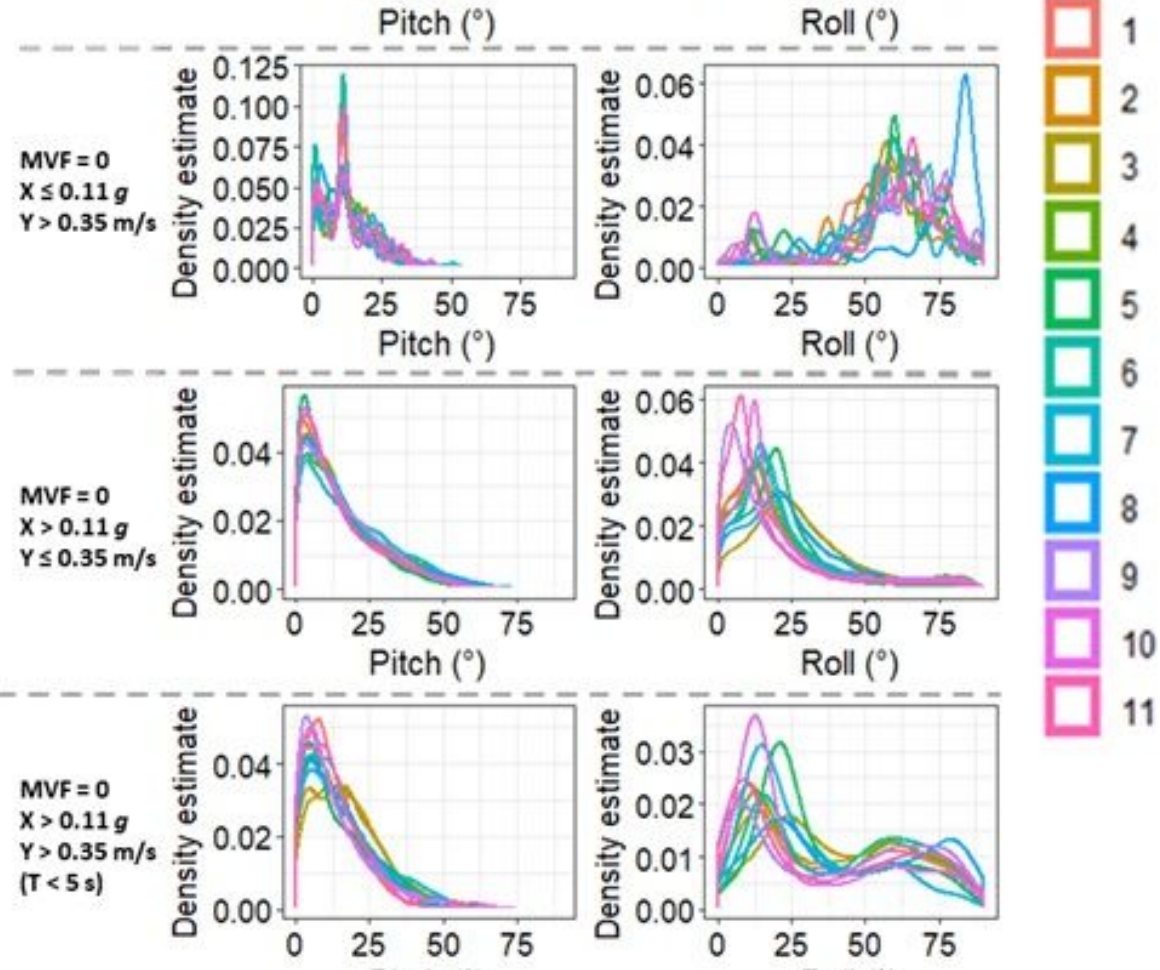

Pitch $\left({ }^{\circ}\right)$

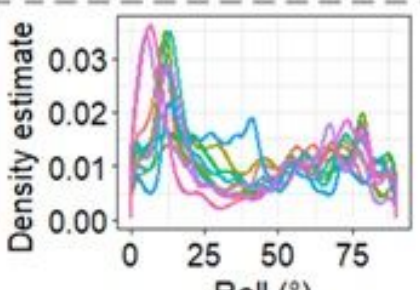

Lion ID

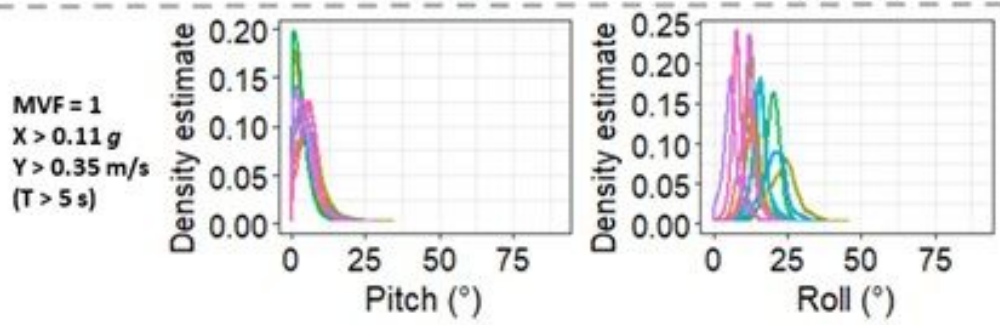

Figure 4

Indices of collar postural offsets per lion, assessed via density estimates of absolute values of; (A) pitch and (B) roll. Plots are facetted row-wise according to 5 scenarios as described to the left of each plot row. The distributions become tighter and more 'bell-shaped' at higher levels of activity 


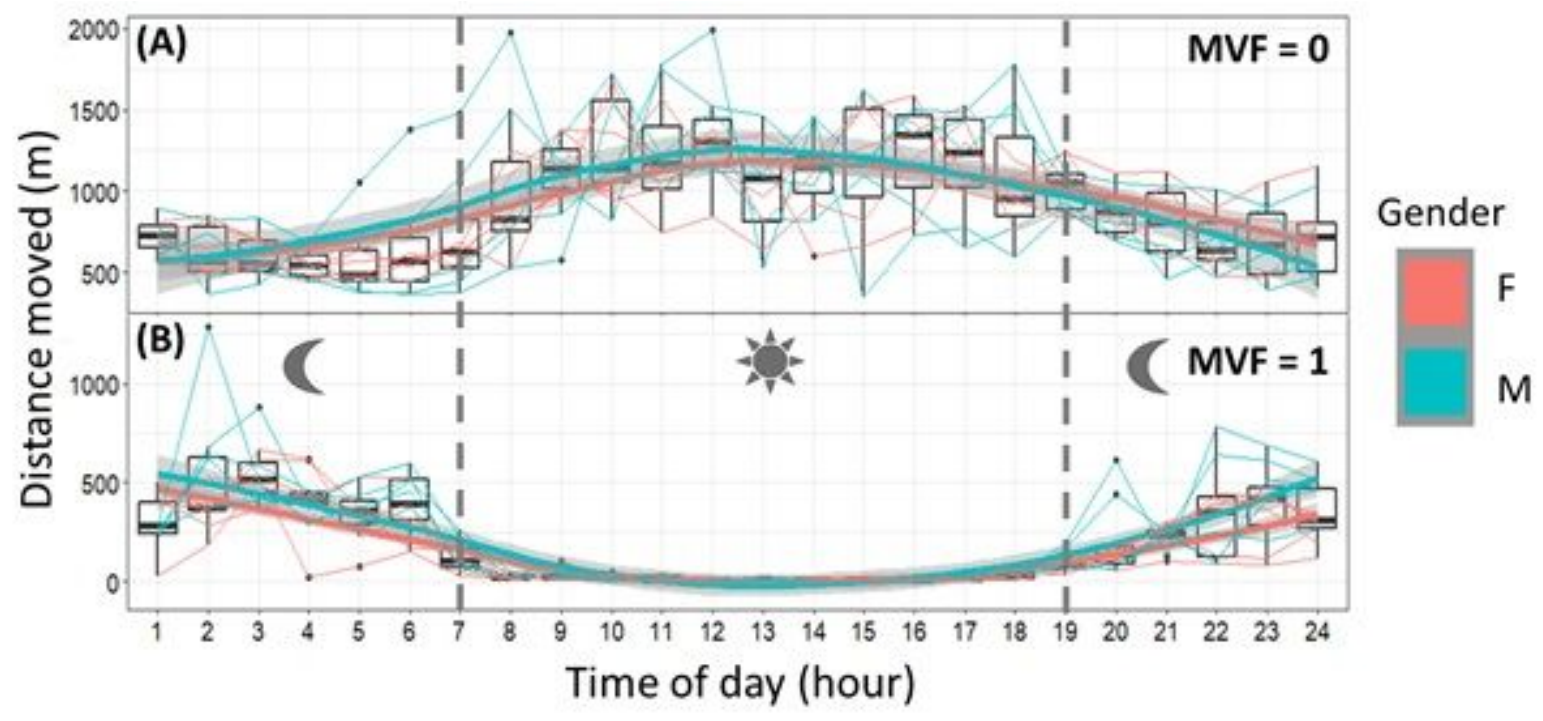

Figure 5

Mean summed distance moved $(\mathrm{m})$ per hour per individual (cf. SI.4 for full description of methods). Each individual's hourly mean is connected across time via a straight-line (coloured according to gender; red = female, blue $=$ male). Plots are fitted with a line of best fit according to gender, using a 'gam smoothing' (grey shading around line represents the $95 \%$ confidence level interval). This procedure was applied independently for non-moving (A) and moving (B). Note the disparity in distance estimates, with nonmoving bouts demonstrating high values during sunlight hours (approximately between 7 am to $7 \mathrm{pm}$ [grey bars]).

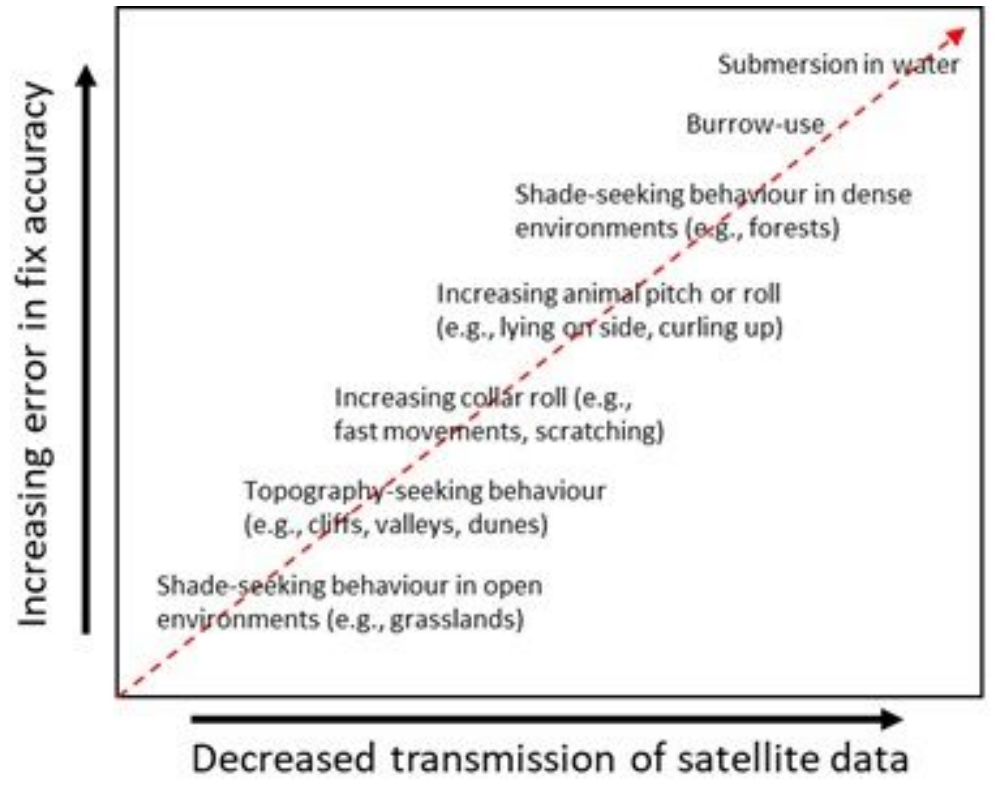

Figure 6

Schematic of the factors related to animal behaviour that can change the quality of GPS fixes.

\section{Supplementary Files}


This is a list of supplementary files associated with this preprint. Click to download.

- SupplementaryMaterial.docx 